\title{
A Practical Method for Detecting Fluff Quality of Fabric Surface Using Optimal Sensing
}

\author{
Shoufeng Jin ${ }^{1}$, Qiangqiang $\mathrm{Lin}^{1}$, Yu Bie ${ }^{2}$, *, Qiurui $\mathrm{Ma}^{3}$, Zhixiong $\mathrm{Li}^{4}$ \\ ${ }^{I}$ Department of Mechanical Engineering, Xi'an Polytechnic University, \\ No. 95, Jinhua North Road, Xincheng District, Xi'an, Shaanxi 710048, China \\ ${ }^{2}$ School of Chemical Engineering, Kunming University of Science and Technology, \\ 727 Jingming South Road, Chenggong District, Kunming, Yunnan 650504, China \\ ${ }^{3}$ College of Fashion and Art of Design, Xi'an Polytechnic University, \\ No. 95, Jinhua North Road, Xincheng District, Xi'an, Shaanxi 710048, China \\ ${ }^{4}$ Department of Marine Engineering, Ocean University of China, \\ No. 238, Songling Road, Tsingdao 266100, China \\ 20070126@kust.edu.cn
}

\begin{abstract}
The raising process has been widely used in manufacturing fabric productions. After raising the surface of the fabric, productions are covered with a fluff layer. The quality of the fabric surface is often valuated by the fluffing type. In order to objectively assess the fluff quality of the fabric surface, an optimal sensing method is proposed in this paper. The fluff contour image was firstly collected by the light-cut imaging device. Then, the fluff region was segmented by the adaptive image segmentation method, the contour coordinates of the fabric were extracted using the freeman chain code and constructed in the form of the binary image. Lastly, a back-propagation neural network (BPNN) was used to learn the relationship between the contour coordinates and the fluff quality. On this basis, a practical fabric fluff detection platform was developed based on the optimal sensing technique. Experimental tests were conducted to evaluate the performance of the proposed method in detecting the fluff quality with four different colours and different fluffing processes. Furthermore, the actual fabric inspection was carried out. The detection correct rate can reach $94.17 \%$, which can meet the practical production requirement.
\end{abstract}

Index Terms-Fabric manufacturing; Machine vision; Artificial intelligence; Optical imaging.

\section{INTRODUCTION}

With the improvement of material quality, the quality requirement on the fabric surface is increasing. The raising process is an essential finishing part for high-quality fabric manufacturing. A fluff layer with a certain thickness and uniform distribution on the fabric surface is generated after the raising processing, which makes the fabric surface soft and enhances the thermal insulation of the fabric [1]. The quality of the fluff on the fabric surface has a great influence on the performance of the fabric productions. Accurately and

Manuscript received 17 September, 2019; accepted 30 January, 2020.

This research was supported by Shan'xi Provincial Natural Science Foundation under grant (No. 2017JM141), Shan'xi Provincial Department of Education Special Research under grant (No. 17JK0334), Qingdao Fundamental Research programme under grant (No. 19-6-2-14-cg), Doctoral Fund of Xi' an Polytechnic University under grant (No. BS1535), and Xi'an Science and Technology Plan programme under grant (No. 201805030YD8CG14(5)). objectively evaluating the quality of the fabric fluff is still a challenging task [2]. At present, the fluff quality relies on manual checking, which causes many problems such as time-consuming, miss checking, and high labour cost. More importantly, the quality index cannot be quantified by manual checking. Therefore, it is crucial to develop automatic detection method for the textile industry [3].

Optimal sensing technique is regarded as an effective tool for automatic fluff detection. Up to today, the optimal detection of fluff mainly focuses on the fabric defects and pilling [4]. Mahajan et al. [4] compared existing methods to provide an important reference for studying fluff detection. Ngan et al. [5] studied various fabric detection methods and described the advantages and disadvantages of these methods. Chen and Feng [6] proposed a method based on two optimal filters for fabric defect detection, which can accurately detect the fabric defects, including colour images. Li et al. [7] developed a multi-channel feature extraction method to detect plain and twill weave patterns in the fabric defects. Kim and Park [8] proposed a method to detect ball pilling mixing. Guan et al. [9] applied the back-propagation neural network (BPNN) to achieve high-precision classification of pilling fabric images. Uyanik et al. [10] studied the relationship between the pilling and fabric knit construction. However, there is a very limited evaluation for the fluffing state of the fleece fabrics. An automatic detection system has not been used in practical manufacturing. In response to the actual needs of the textile industry, it is necessary to develop a practical method for detecting fluff quality of fabric surfaces.

In order to meet the actual needs of the textile industry, this paper proposes an optimal sensing method based on BPNN to detect the quality of fluff fabrics. The BPNN has been widely used in various fields owing to its superior learning ability [11]. BPNN is able to complete nonlinear fitting and complex classification problems [12]. Therefore, the BP neural network can be applied to determining whether the surface of the fabric is qualified. In this paper, for this gaol, both experimental and industrial images were used to build a BPNN detection system based on the principle of light-cut 
imaging. The raining of the BPNN was achieved by extracting the coordinate information of the upper edge of the fabric using the fluff-related images. The detection results showed the effectiveness of the proposed method.

\section{PRACTICAL SOLUTION}

The raising process is a finishing process of the fabric productions, which directly affects the style and quality of the fabrics. Fabric fluffing is a dynamic change process. As shown in Fig. 1(a), the raising roller wrapped with the hairpin cloths is circumferentially distributed on the cylinder, and the raised fabric is close to the cylinder due to the two traction rollers between the cloths. There is a speed difference in the cloth rollers, and the fabric has a certain tension on the surface of the raising roller. The raising roller rotates with the cylinder. Due to the relative movement between the raising roller and the raised fabric, the raising action is achieved. The resulting raised fabrics are shown in Fig. 1(b). There are four typical fabrics provided by a fabric company, among them Sample 1, Sample 2, and Sample 3 are qualified, while Sample 4 is the unqualified fabric. The need of enterprises is to assess different types of fabrics.

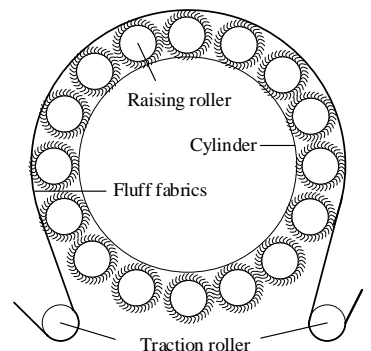

(a)

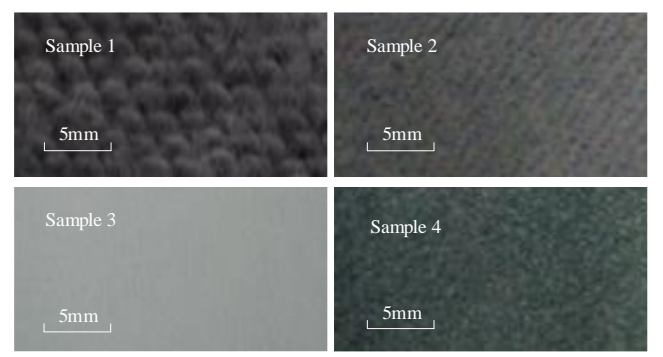

(b)

Fig. 1. Images of fabric fluff: (a) Process fabric fluff; (b) Typical samples provided by enterprises.

Dense fluff has a certain thickness on the surface of the fabric. Taking the fabric type in Fig. 1(b) as an example, in order to obtain the thickness and distribution of the fluff layer, a tangential image of the fabric is collected by a test bench using the principle of light-cut imaging. The actual acquisition equipment is shown in Fig. 2.

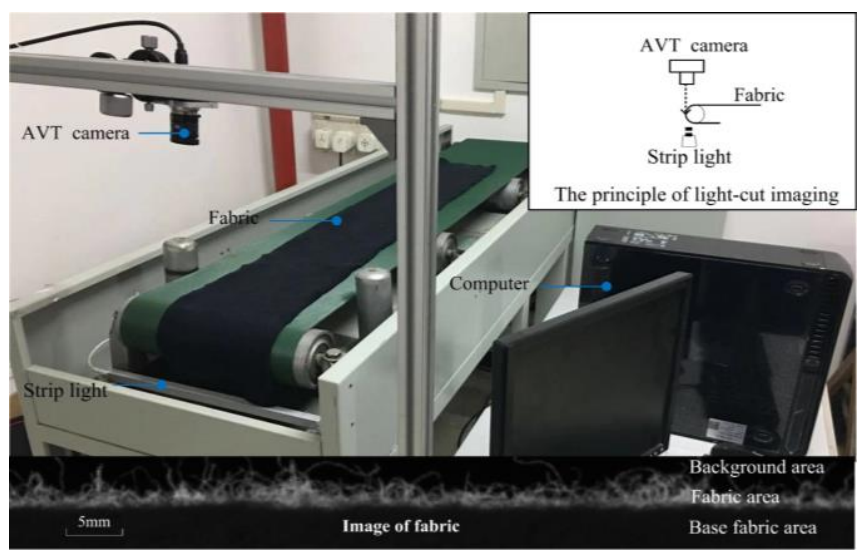

Fig. 2. The practical fabric detection optimal system.

In this practical detecting system, the Matlab software is used as the experimental environment, and the neural network toolbox is employed to complete the training and implementation of the BPNN. The computer processor model is Intel(R) Core(TM) i5-4440@3.10 GHz, and the installed memory is $8 \mathrm{G}$. The camera and the strip light source are placed on both sides of the fabric, respectively. The strip light source is placed directly under the raising roller, so that the light is tangent to the edge of the axial contour of the roller and the background light image can be used to avoid the texture and color characteristics of the fabric surface. Highlighting the contour of the fluff edge is considered. The fabric is coated on the surface of the roller and rotates at a constant speed with the roller. At the shooting position of the optimal sensors, the thickness of the fluff on the surface of the fabric is detectable under the tension of the roller. The image size obtained from the optimal device is $1024 \times 1024$ pixels, and the image is cropped to the area of interest. A valid area of $200 \times 840$ pixels is shown at the bottom of Fig. 2, which is mainly divided into one background area, one fabric area, and one base fabric area. In order to obtain a clear image of the fabric area and reduce the influence of the background on the fluff profile, the black colour is used for the background. The fabric area can be clearly separated from the background by the strip light, so as to obtain the required features.

The image is segmented to extract the features of the fluff region after separating the background region from the base fabric. From the grayscale histogram distribution shown in Fig. 3, one can note that the image is adaptively segmented by the maximum inter-class variance method. The upper edge contour of the image reflects the thickness and distribution of the fabric. The contour features of the upper edge are extracted using the freeman chain code. Lastly, the BPNN is used to establish the detection model using the contour features. Figure 4 describes the proposed method.

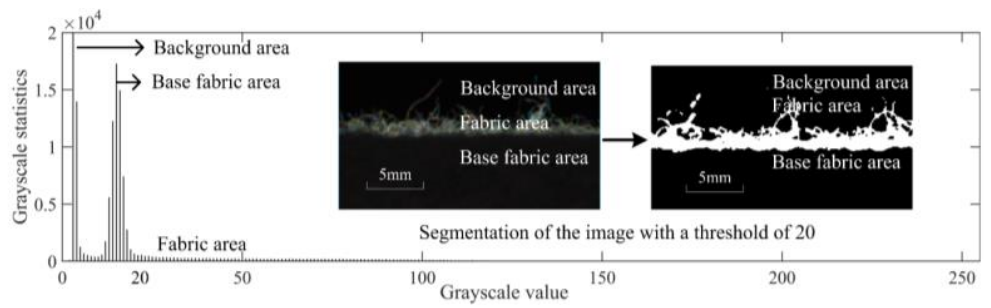

Fig. 3. Grayscale histogram distribution. 


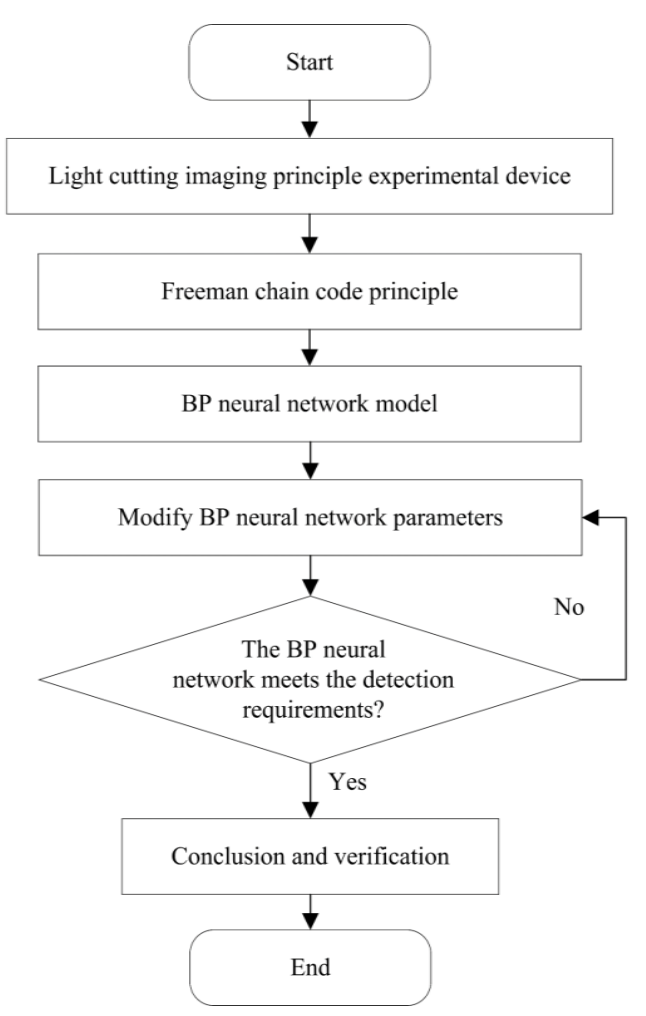

Fig. 4. The proposed method for fluff quality detection.

\section{EXPERIMENTAL AND INDUSTRIAL EVALUATION}

\section{A. Feature Extraction}

The chain code technology is originally applied to linear data programming. In this paper, the freeman chain code [13] is used to extract the image features as shown in Fig. 5. There are two types of the chain code, one has eight directions and the other has four directions. In the generated contour calculation diagram, the unidentified position pixel is given a 0 , while the contour edge pixel is given a 1 . By doing so, it can ensure the formation of an eight-neighbour or four-neighbour image.

Accurate extraction of the contour features of the fabric edge is the premise to establish the BPNN detection model. The eight-neighbour chain code is used to extract the feature points of the fabric fluff edge. The calculation process is described as follows [13].

1. Calculate the size of the edge of the extracted contour (defined as $M \times N)$ and, then, generate a matrix with the size of $(M+2, N+2)$, and embed the edge contour into the matrix.

2. Find the coordinates of the first edge pixel as the starting point for the edge search. Then, search for the remaining 7 directions and find their coordinates.

3 . If the remaining 7 directions are all 0 , terminate the search and return the coordinate values. Then, the edge coordinates of the fabric profile can be extracted.

Table I shows a portion of the extracted coordinate points of a fabric image acquired from the industrial application (see Fig. 6). It can be seen from Fig. 6 that the edge contour of the fabric extracted by the proposed method is basically matched with the upper edge of the fabric and can reflect the edge contour features of the fluff.

TABLE I. PART OF THE COORDINATE POINTS.

\begin{tabular}{|c|c|c|c|c|c|c|c|c|c|}
\hline No. & \multicolumn{9}{|c|}{ Upper edge feature point } \\
\hline 1 & 98 & 98 & 99 & 99 & 99 & 99 & \multirow{5}{*}{$\cdots$} & 98 & 97 \\
\hline 2 & 90 & 91 & 92 & 93 & 94 & 94 & & 94 & 93 \\
\hline 3 & 87 & 87 & 86 & 85 & 84 & 83 & & 83 & 83 \\
\hline 5 & 99 & 99 & 100 & 101 & 101 & 100 & & 100 & 100 \\
\hline 6 & 91 & 92 & 93 & 93 & 93 & 93 & & 93 & 93 \\
\hline
\end{tabular}

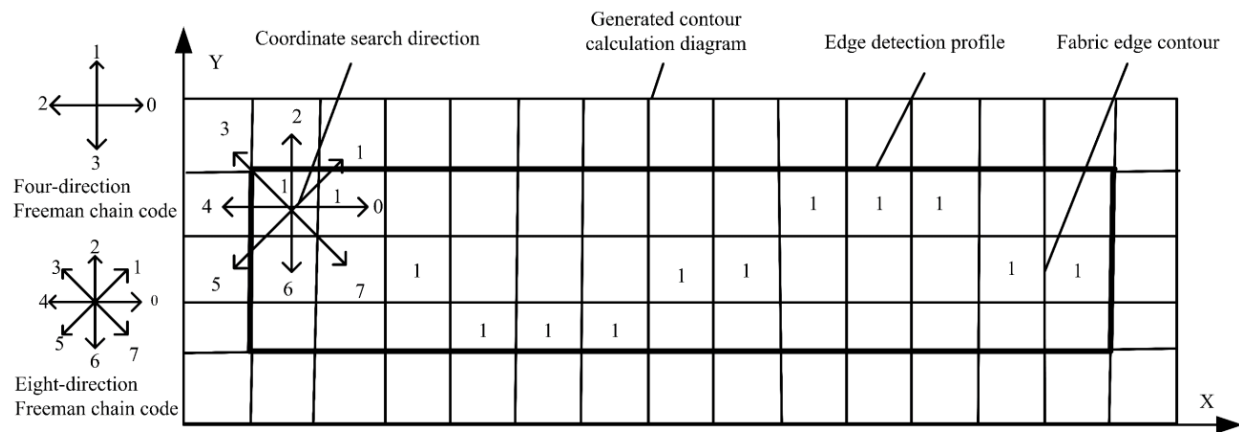

Fig. 5. Contour coordinates calculation.

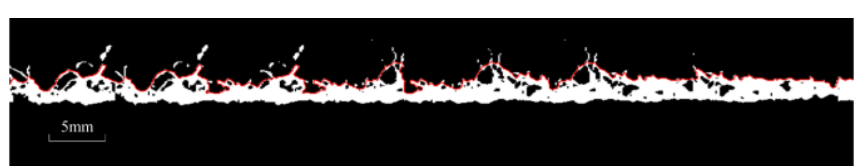

Fig. 6. An image of the fabric contour acquired form industry.

\section{B. BPNN Detection Model}

BPNN is usually a three-layer network [14]. The extracted fabric contour coordinates are taken as the inputs of the BPNN, and the target outputs are [1,0], where 1 denotes the qualified fluff, while 0 denotes the unqualified fluff.

In the experimental study, a total of 435 fabric images were collected, of which 225 were qualified fluff, while 210 were unqualified fluff. Figure 7 illustrates eight fabric images, where the label $U$ means unqualified and label $Q$ means qualified fluff. Figs. 7(b)-7(e) show the unrecognized fabrics, whose BPNN target outputs are 0, while Figs. 7(f)-7(i) are the good fabrics, whose BPNN target outputs are 1.

Firstly, the freeman chain code was applied to all 435 images to extract the contour features of the fluff. For each image, 840 contour coordinates were obtained. As a result, the structure of the BNPP was $840 \times 10 \times 1$, where 840 was the neuron number of the input layer, 10 was the neuron number of the hidden layer, and 1 was the neuron number of the output layer. The steepest descent method was used to train the BPNN. The activation function may influence the model performance. In this study, different activation functions were compared in the BPNN training process, including the 
Log-Sigmoid (logsig), Tan-Sigmoid (tansig), and linear functions (purelin). Figure 9 shows the comparison results.

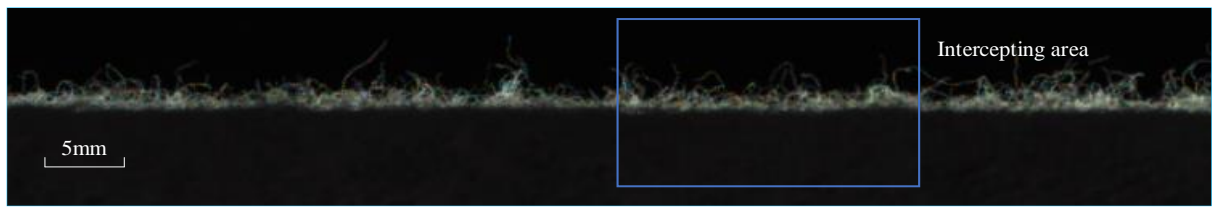

(a)

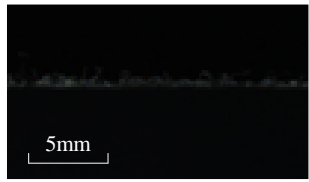

(b)

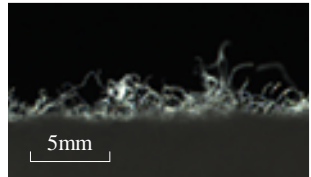

(f)

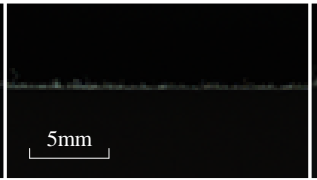

(c)

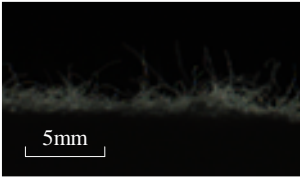

$(\mathrm{g})$

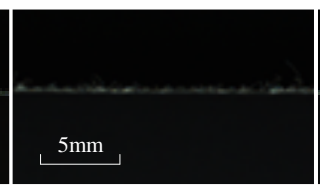

(d)

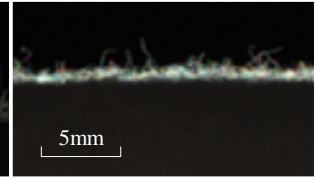

(h)

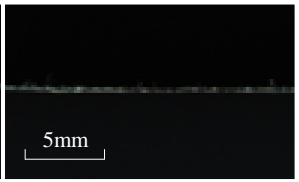

(e)

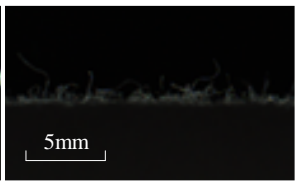

(i)

Fig. 7. A portion of the original images: (a) The original image; (b) 1-U-0 ;(c) 2-U-0; (d) 3-U-0; (e) 4-U-0; (f) 5-Q-1; (g) 6-Q-1; (h) 7-Q-1; (i) 8-Q-1. (Note: figure No. (b) to (i) expressed by $a-b-c$, where $a$ is sample number; $b$ is the quality of the fabric, divided into qualified and unqualified, $\mathrm{U}$ is unqualified and $\mathrm{Q}$ is qualified; $c$ is label, divided into 0 and 1,0 is unqualified and 1 is qualified. For example,1-U-0 means sample1 is unqualified and label is 0 )

It can be seen from Fig. 8(a) that, when the tansig activation function was used for both the hidden and output layers, the detection accuracy was $93.12 \%$, while for the other three combinations the detection accuracy was below $70 \%$.

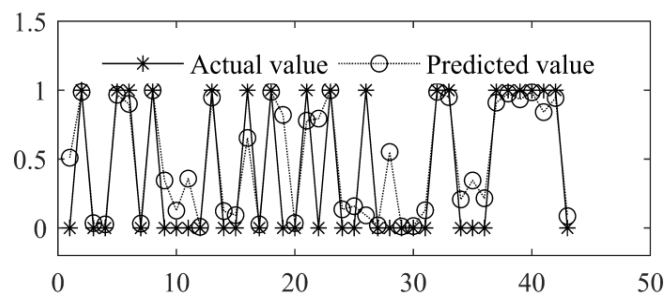

(a)

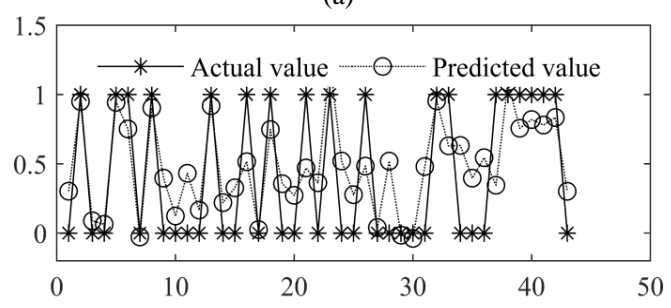

(b)

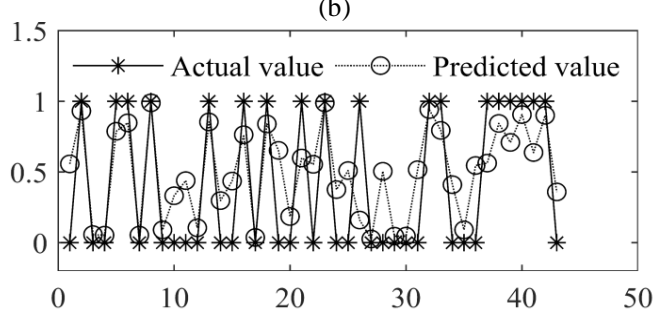

(c)

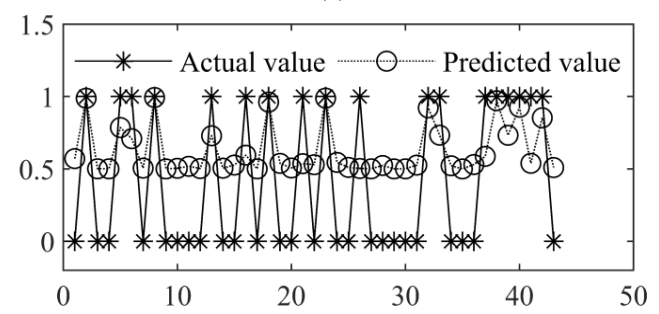

(d)

Fig. 8. Effect of different combination of the activation functions: (a) Tansig-tansig; (b) Logsig-purelin; (c) Logsig-tansig; (d) Tansig-logsig.
Moreover, the effect of the neuron number in the hidden layer on the training performance of the BPNN was investigated. Figure 9 depicts the analysis results.

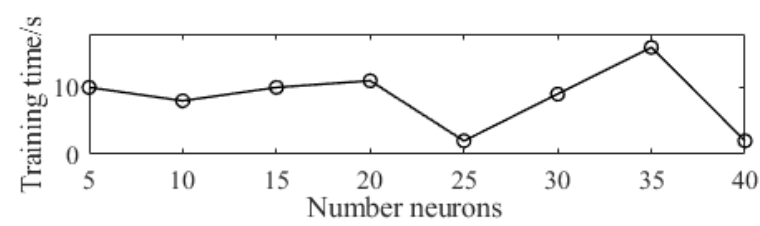

(a)

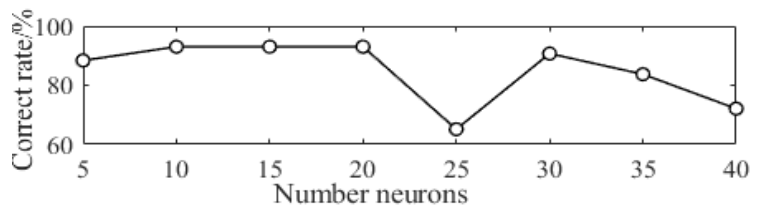

(b)

Fig. 9. Effect of the neuron number in the hidden layer: (a) training time; (b) correct rate.

As can be seen from the Figure, when the neuron number was between 5 and 20, the detection rate presented a rising trend, but the training time trended to increase; when the neuron number was between 20 and 25 , the correct rate significantly decreased, so that the BPNN terminated the training resulting in the decrease of the training time; when the neuron number increased further, fluctuations occurred in both the training time and correct rate. In order to ensure high accuracy and low training time, the number of neurons in the hidden layer chose 10 in this study.

Table II provides the BPNN test results. As can be seen in the table, the proposed method is able to identify the four different fabric fluffs as shown in Fig. 1. The correct rate for each category is beyond $91 \%$ and the highest rate is $100 \%$ for Category 3. Because the contour coordinates of the upper edge of the images were extracted as the features, the proposed optimal sensing method is insensitive to the colour of the images. As a result, this newly proposed method is applicable to various fabric images. 
TABLE II. TESTING RESULTS.

\begin{tabular}{|c|c|c|c|}
\hline $\begin{array}{c}\text { Image } \\
\text { types }\end{array}$ & Test samples & $\begin{array}{c}\text { Correct } \\
\text { number }\end{array}$ & Correct rate \\
\hline Category 1 & 50 & 47 & $94.00 \%$ \\
\hline Category 2 & 50 & 47 & $94.00 \%$ \\
\hline Category 3 & 74 & 74 & $100 \%$ \\
\hline Category 4 & 169 & 155 & $91.72 \%$ \\
\hline
\end{tabular}

\section{Industrial Application}

Furthermore, the proposed BPNN-based optimal sensing system was installed in an industrial machine for fluff quality detection. The fabrics shown in Fig. 1 were tested. The detection accuracy of the three qualified fabrics was $96.55 \%$, while the accuracy of the unqualified fabric was $94.17 \%$. As a result, the proposed method can meet the actual production inspection needs, and hence can be used in industrial applications.

\section{CONCLUSIONS}

Based on the requirements of fabric manufacturer, this paper proposes an intelligent optimal sensing method to detect the fluff quality of the fabric productions. Firstly, an experimental platform was built using the light-cut imaging. The images of four fabrics provided by the manufacturer were processed using the freeman chain code to extract the edge coordinates as the input features of a BPNN model. In the training process, the parameters of the BPNN were optimized. The experimental results show that the training time of the BPNN was less than $20 \mathrm{~s}$ and the detection rate was above $90 \%$ for the four fabrics. The industrial investigation results demonstrate an average accuracy of $95.36 \%$. As a result, the proposed method can be applied to industry to save labour costs and improve production efficiency.

\section{CONFLICTS OF INTEREST}

The authors declare that they have no conflicts of interest.

\section{REFERENCES}

[1] M. Shabbir, L. Rather, and F. Mohammad, "Economically viable
UV-protective and antioxidant finishing of wool fabric dyed with targets erect a flower extract: Valorization of marigold", Industrial Crops and Products, vol. 119, pp. 277-282, 2018. DOI 10.1016/j.indcrop.2018.04.016.

[2] A. Guha, C. Amarnath, S. Pateria et al., "Measurement of yarn hairiness by digital image processing", Journal of the Textile Institute, vol. 101, no. 3, pp. 214-222, 2010. DOI: 10.1080/00405000802346412.

[3] D. Schneider and D. Merhof, "Blind weave detection for woven fabrics", Pattern Analysis and Applications, vol. 18, no. 3, pp. 725-737, 2015. DOI: 10.1007/s10044-014-0403-9.

[4] P. M. Mahajan, S. R. Kolhe, and P. M. Patil, "A review of automatic fabric defect detection techniques", Advances in Computational Research, vol. 1, no. 2, pp. 18-29, 2009.

[5] H. Ngan, G. Pang, and N. Yung, "Automated fabric defect detection A review", Image and Vision Computing, vol. 29, no. 7, pp. 442-458, 2011. DOI: 10.1016/j.imavis.2011.02.002.

[6] Z. Chen and X. Feng, "The design of optimal real Gabor filters and their applications in fabric defect detection", Coloration Technology, vol. 131, no. 4, pp. 279-287, 2015. DOI: 10.1111/cote.12154.

[7] C. Li, C. Liu, G. Gao et al., "Robust low-rank decomposition of multi-channel feature matrices for fabric defect detection", Multimedia Tools and Applications, vol. 78, no. 6, pp. 7321-7339, 2019. DOI 10.1007/s11042-018-6483-6.

[8] S. Kim and C. Park, "Evaluation of fabric pilling using hybrid imaging methods", Fibers and Polymers, vol. 7, no. 1, pp. 57-61, 2006. DOI: 10.1007/BF02933603.

[9] S. Guan, W. Li, J. Wang et al., "Objective evaluation of fabric pilling based on data-driven visual attention model", International Journal of Clothing Science and Technology, vol. 30, no. 2, pp. 210-221, 2018. DOI: 10.1108/IJCST-04-2017-0042.

[10] S. Uyanik and M. Topalbekiroglu, "The effect of knit structures with tuck stitches on fabric properties and pilling resistance", The Journal of the Textile Institute, vol. 108, no. 9, pp. 1584-1589, 2017. DOI: 10.1080/00405000.2016.1269394.

[11] A. Glowacz, W. Glowacz, Z. Glowacz et al., "Early fault diagnosis of bearing and stator faults of the single-phase induction motor using acoustic signals", Measurement, vol. 113, pp. 1-9, 2018. DOI: 10.1016/j.measurement.2017.08.036.

[12] A. Glowacz, "Fault diagnosis of single-phase induction motor based on acoustic signals", Mech. Sys. Sig. Process, vol. 117, pp. 65-80, 2019. DOI: 10.1016/j.ymssp.2018.07.044.

[13] K. Prasad and P. Aithal, "A study on fingerprint hash code generation based on MD5 algorithm and freeman chain code", Int. J. Comput. Research Develop., vol. 3, no. 1, pp. 13-22, 2018. DOI 10.5281/zenodo.1144555.

[14] Z. Li, Z. Zhang, J. Shi, and D. Wu, "Prediction of surface roughness in extrusion-based additive manufacturing with machine learning", Robotics and Computer-Integrated Manufacturing, vol. 57, pp. 488-495, 2019. DOI: 10.1016/j.rcim.2019.01.004 